\title{
Prime Factors of Cyclotomic Class Numbers
}

\author{
By D. H. Lehmer
}

\begin{abstract}
Let $p$ be an odd prime. The "first factor" $h^{*}(p)$ of the class number of the field of $p$ th roots of unity has been the subject of many investigations beginning with Kummer (1861). In the present paper it is shown how the theory of a function introduced by T. A. Pierce (1917) can be used to find the prime factors of $h^{*}(p)$.
\end{abstract}

1. Introduction. Let $p$ be an odd prime with a primitive root $g$. Let $g^{n} \equiv$ $g_{n}(\bmod p)\left(0<g_{n}<p\right)(0 \leqslant n<p-1)$. Denote by $F=F_{p}$ the polynomial

$$
F_{p}(x)=\sum_{n=0}^{p-2} g_{n} x^{n}
$$

Finally, let $\theta=\exp \{2 \pi i /(p-1)\}$. Then $h^{*}(p)$, the so-called first factor of the number of classes of ideals in the field generated by $\exp \{2 \pi i / p\}$, is given by Kummer's formula $[3$, p. 358 , formula (5.6)]

$$
(2 p)^{(p-3) / 2} h^{*}(p)=\left|\prod_{\nu=0}^{(p-3) / 2} F_{p}\left(\theta^{2 \nu+1}\right)\right| .
$$

In Kummer's original paper [1] the formula appears without absolute value signs. If these are omitted, it is necessary to include a minus sign in (2) above, as will be shown below. It is our purpose to show in an elementary way how the theory of Pierce's function, as developed in [2], can be used to sort out the prime factors of $h^{*}(p)$ into arithmetic progressions so as to render feasible the factorization of $h^{*}(p)$ for quite large values of $h^{*}$.

2. Notation and Lemmas. Let $M=2^{\lambda} \omega, \omega$ odd, be any positive integer and let $Q_{k}(x)$ be the cyclotomic polynomial whose roots are the primitive $k$ th roots of unity. Let $\Omega_{M}(x)$ be the monic polynomial whose roots are the distinct odd powers of $\rho=\exp \{2 \pi i / M\}$.

Lemma 1. $\Omega_{M}(x)=\Pi_{\delta \mid \omega} Q_{M / \delta}(x)$.

Proof. In case $M$ is odd, so that $M=\omega$, the lemma becomes the familiar identity

$$
\prod_{d \mid M} Q_{d}(x)=x^{M}-1
$$

In case $M$ is even we have

Received August 30, 1976; revised September 20, 1976.

AMS (MOS) subject classifications (1970). Primary 12A35, 12A50, 12-04, $10 \mathrm{~A} 35$. 


$$
\Omega_{M}(x)=\prod_{n=1 ; n \text { odd }}^{M}\left(x-\rho^{n}\right)=\prod_{\delta \mid \omega} \prod_{(t, M / \delta)=1}\left(x-\rho^{t \delta}\right)=\prod_{\delta \mid \omega} Q_{M / \delta}(x)
$$

We define Pierce's function $Q_{k}^{*}(P)$ of the polynomial $P$ by

$$
Q_{k}^{*}(P)=\prod_{i=1}^{r} Q_{k}\left(\beta_{i}\right)
$$

where $\beta_{i}$ are the roots of $P$. When $P$ is monic with integer coefficients, it is clear that $Q_{k}^{*}(P)$ is an integer, being a symmetric function of the roots of $P$.

Before proceeding further, we give a variant of Kummer's formula (2) which has two advantages: (a) it is analytic, (b) it replaces $F_{p}$ by a monic polynomial.

LEMMA 2. Let $G_{p}(x)$ be the polynomial

$$
G_{p}(x)=\sum_{n=0}^{p-2} g_{n} x^{p-n-2}
$$

Then

$$
(2 p)^{(p-3) / 2} h^{*}(p)=\prod_{\nu=0}^{(p-3) / 2} G_{p}\left(\theta^{2 \nu+1}\right) .
$$

Proof. Comparing (4) with (1), we see that

$$
G_{p}(x)=x^{p-2} F_{p}(1 / x)
$$

and that

$$
\left|G_{p}\left(\theta^{2 \nu+1}\right)\right|=\left|\theta^{(p-2)(2 \nu+1)}\right|\left|F_{p}\left(\theta^{p-2-2 \nu}\right)\right|=\left|F_{p}\left(\theta^{2 \lambda+1}\right)\right|
$$

where

$$
\lambda=(p-3) / 2-\nu
$$

Hence the product in (5) does not differ in absolute value from that in (2). It remains to show that it is positive.

If we compare $\theta^{2 \nu+1}$ with $\theta^{2 \lambda+1}$, where $\lambda$ is defined by (6), we see that they are complex conjugates and so the corresponding factors of $(5), G_{p}\left(\theta^{2 \nu+1}\right)$ and $G_{p}\left(\theta^{2 \lambda+1}\right)$, have a positive product to contribute to (5) as long as $\nu$ and $\lambda$ are distinct. If they are equal, their value is $(p-3) / 4$, which can happen only when $p \equiv-1(\bmod 4)$. It remains to consider this case in which $\theta^{2 v+1}=-1$. To prove the lemma it suffices, then, to show that $G_{p}(-1)$ is positive. In fact, more is true, namely if $p \equiv 3(\bmod 4)$

$$
G_{p}(-1)=p h
$$

where $h$ denotes the class number of the imaginary quadratic field $K(\sqrt{-p})$. We have only to note that

$$
G_{p}(-1)=\sum_{n=0}^{p-2} g_{n}(-1)^{p-n-2}=-\sum_{\nu=1}^{p-1} \nu\left(\frac{\nu}{p}\right)
$$


since the $g$ 's with even subscripts are the quadratic residues of $p$. But it is well known that (see, for example, [3, p. 344, formula (4.3)])

$$
\sum_{\nu=1}^{p-1} \nu\left(\frac{\nu}{p}\right)=-p h
$$

so (7) follows and the lemma is proved. This also gives a simple proof of the following well-known [6]

Corollary. If $p \equiv 3(\bmod 4)$, then $h^{*}(p)$ is divisible by $h$.

\section{First Factorization Theorem.}

THEOREM 1. Let $p$ be an odd prime and let $p-1=2^{\lambda} \omega$ where $\omega$ is odd. Then the right-hand member of

$$
(2 p)^{(p-3) / 2} h^{*}(p)=(-1)^{(p-1) / 2} \prod_{d \mid \omega} Q_{2}^{*} \lambda_{d}\left(G_{p}\right)
$$

is a factorization into rational integers.

Proof. The degree of $\Omega_{p-1}(x)$ is seen to be $(p-1) / 2$ while that of $G_{p}(x)$ is $p$ - 2. The right-hand side of $(5)$ is the product of $G_{p}(x)$ taken over the roots of $\Omega_{p-1}(x)$ and is thus the resultant

$$
\begin{aligned}
R\left(G_{p}, \Omega_{p-1}\right) & =(-1)^{(p-2)(p-1) / 2} R\left(\Omega_{p-1}, G_{p}\right) \\
& =(-1)^{(p-1) / 2} \prod_{i=1}^{p-2} \Omega_{p-1}\left(\alpha_{i}\right) \quad\left(G_{p}\left(\alpha_{i}\right)=0\right) \\
& =(-1)^{(p-1) / 2} \prod_{d \mid \omega} Q_{2 \lambda_{d}}^{*}\left(G_{p}\right)
\end{aligned}
$$

by Lemma 1 . Since $G_{p}$ is monic with integer coefficients the $Q^{*}$ 's are integers.

This theorem allows us to "divide and conquer" the problem of factoring $h^{*}(p)$ by considering separately the prime factors of the $Q^{*}$ 's.

4. Second Factorization Theorem. Of course, the product on the right of (8) must contain at least $(p-3) / 2$ factors 2 and $p$, and we show in Section 5 how these can be removed automatically in obtaining a more efficient variant of (8). Other prime factors of $Q_{2}^{*} \lambda_{d}\left(G_{p}\right)$ may divide $d$ and are called intrinsic factors and are discussed in Sections 8 and 9 . They are easily discovered and removed. The remaining prime factors of $Q_{2 \lambda_{d}}^{*}$ are called characteristic. To facilitate their discovery we use the following lemma.

Leмma 3. Let $\pi^{k}$ be the highest power of a characteristic prime $\pi$ dividing $Q_{n}^{*}(p)$. Let $\mu$ be the least positive exponent for which $\pi^{\mu} \equiv 1(\bmod n)$. Then $\mu \mid k$.

Proof. A proof of this fundamental result from the theory of Pierce functions is found in [1].

THEOREM 2. Let $P_{d}=q_{1} q_{2} \cdots q_{t}$ be the product of all the characteristic factors of $Q_{2 \lambda_{d}}^{*}\left(G_{p}\right)$ into distinct powers of odd primes. Then

$$
q_{i} \equiv 1 \quad\left(\bmod 2^{\lambda} d\right) \quad(i=1(1) t) .
$$


Proof. Using Lemma 3 with $\pi^{k}=q_{i}, n=2^{\lambda} d, P=G_{p}$ and writing $k=\mu j$, we have at once

$$
q_{i}=\pi^{k}=\left(\pi^{\mu}\right)^{j} \equiv 1^{j}=1 \quad\left(\bmod 2^{\lambda} d\right) .
$$

To search for the prime factors of $P_{d}$, we therefore try as divisors of $P_{d}$ only the numbers in the arithmetic progression $2^{\lambda} d x+1(x=1,2,3, \ldots)$. The first such divisor is either a prime or a power of a prime. After removing all such factors below some limit, an attempt can be made to represent the cofactor as $a^{2}-b^{2}$. In this case $a$ is restricted to one case modulo $2^{2 \lambda-1} d^{2}$.

5. Simplification of Character Sums. We now develop a practical method of computing an isolated value of $Q_{2 \lambda_{d}}^{*}\left(G_{p}\right)$. This involves four lemmas and the following notation.

$$
\begin{aligned}
& p \text { is an odd prime. } \\
& g \text { is a primitive root of } p . \\
& p-1=e f \text { where } f \text { is odd. } \\
& \tau=e /\left(e, \text { ind }_{g} 2\right) . \\
& \alpha=\exp \{2 \pi i / e\} . \\
& \chi(k)=\chi_{e}(k)=\alpha^{\text {ind }} k \quad \quad\left(\chi_{e}(0)=0\right) . \\
& M_{e}(p)=\sum_{k=1}^{p-1} k \chi_{e}(k) . \\
& m_{e}(p)=\sum_{k=1}^{(p-1) / 2} \chi_{e}(k) .
\end{aligned}
$$

Lemma 4. Let $r$ be any integer and let $(r, e)=\delta$ so that $e=\delta e_{1}$. Then

$$
\prod_{t \leqslant e ;(t, e)=1}\{x-\exp (2 \pi i r t / e)\}=\left\{Q_{e_{1}}(x)\right\}^{\phi(e) / \phi\left(e_{1}\right)}
$$

where $\phi(n)$ is Euler's totient function.

Proof. The left member of (9) is a polynomial $\psi(x)$ of degree $\phi(e)$ which is monic and has for roots all the primitive $e_{1}$ th roots of unity each with the same multiplicity $\nu$, say. That is, $\psi(x)=\left\{Q_{e_{1}}(x)\right\}^{\nu}$. Taking the degrees of both sides of this identity, we have $\phi(e)=\nu \phi\left(e_{1}\right)$, which proves the lemma.

LEMma 5. The norm of $2-\chi(2)$ in the cyclotomic field of the eth roots of unity is

$$
N_{e}\left(2-\chi_{e}(2)\right)=\left\{Q_{\tau}(2)\right\}^{\phi(e) / \phi(\tau)}
$$

Proof. Set $r=\operatorname{ind}_{g} 2$ and $x=2$ in Lemma 4.

Lemma 6. $\left\{2-\chi_{e}(2)\right\} M_{e}(p)=-p m_{e}(p)$.

Proof. First we note that $\chi_{e}(-1)=-1$. In fact

$$
\chi_{e}(-1)=\chi_{e}(p-1)=\alpha^{\text {ind }(p-1)}=\alpha^{(p-1) / 2}=\exp \{\pi i(p-1) / e\}=(-1)^{f}=-1 \text {. }
$$

Now let $M^{\prime}$ denote the half sum 


$$
M^{\prime}=M_{e}^{\prime}(p)=\sum_{k<p / 2} k \chi_{e}(k)
$$

Then

$$
\begin{aligned}
M_{e}(p)-M^{\prime} & =\sum_{r<p / 2}(p-r) \chi_{e}(p-r) \\
& =p \chi_{e}(-1) m_{e}(p)-\chi_{e}(-1) M^{\prime}
\end{aligned}
$$

Hence

$$
M_{e}(p)=-p m_{e}(p)+2 M^{\prime}
$$

On the other hand,

$$
\begin{aligned}
M_{e}(p) & =\sum_{k<p / 2}\left\{2 k \chi_{e}(2 k)+(2 k+1) \chi_{e}(2 k+1)\right\} \\
& =2 \chi_{e}(2) M^{\prime}+\sum_{k<p / 2}(p-2 k) \chi_{e}(p-2 k)
\end{aligned}
$$

or

$$
\chi_{e}(2) M_{e}(p)=4 M^{\prime}-p m_{e}(p) .
$$

Multiplying (10) by 2 and subtracting from (11) gives the lemma.

THEOREM 3.

$$
Q_{e}^{*}\left(G_{p}\right)=(-1)^{\phi(e)} p^{\phi(e)} N_{e}\left(m_{e}(p)\right) /\left\{Q_{\tau}(2)\right\}^{\phi(e) / \phi(\tau)} .
$$

Proof. By definition (3), we have

$$
\begin{aligned}
Q_{e}^{*}=Q_{e}^{*}\left(G_{p}\right) & =(-1)^{\phi(e)} R\left(G_{p}, Q_{e}\right)=(-1)^{\phi(e)} \prod_{t<e ;(t, e)=1} G_{p}\left(\alpha^{t}\right) \\
& =(-1)^{\phi(e)} \prod_{t<e ;(t, e)=1} \sum_{n=1}^{p-1} g_{n} \alpha^{t(p-n-2)} \\
& =(-1)^{\phi(e)} \prod_{t<e ;(t, e)=1} \alpha^{t(p-2)} \prod_{t<e ;(t, e)=1} \sum_{n=1}^{p-1} g_{n} \alpha^{-t n} \\
& =\prod_{t<e ;(t, e)=1} \sum_{n=1}^{p-1} g_{n} \alpha^{t n}=\prod_{t<e ;(t, e)=1} \sum_{k=1}^{p-1} k \chi_{e}(k) .
\end{aligned}
$$

That is, $Q_{e}^{*}\left(G_{p}\right)=N_{e}\left(M_{e}(p)\right)$. By Lemmas 5 and 6 we have the theorem.

We now define a new exponential sum $W_{e}(p)$ by

$$
W_{e}(p)=W_{e}(p, t)=\sum_{n=1}^{(p-1) / 2}\left\{\epsilon_{n}-\epsilon_{n-1}\right\} \alpha^{n t}
$$

$$
\text { where } \epsilon_{n}= \begin{cases}1 & \text { if } g_{n}<p / 2 \\ 0 & \text { otherwise }\end{cases}
$$


Thus the coefficients of $W_{e}$ are \pm 1 or 0 .

Lemma 7. $(1-\alpha) m_{e}(p)=2 W_{e}(p, 1)$.

Proof. For typographic simplicity, we write $p^{\prime}$ for $(p-1) / 2$. Since

$$
\alpha^{p^{\prime}}=\left(\alpha^{e / 2}\right)^{f}=(-1)^{f}=-1
$$

and we have $g_{n+p^{\prime}} \equiv g^{p^{\prime}} g_{n} \equiv-g_{n}(\bmod p)$, then $g_{n+p^{\prime}}=p-g_{n}$ so that $\epsilon_{p^{\prime}+n}=1-$ $\epsilon_{n}$. In what follows the summation index $v$ ranges over $0 \leqslant v \leqslant(p-3) / 2$. From the above we can write

$$
\begin{aligned}
m_{e}(p) & =\sum_{k=1}^{p^{\prime}} \alpha^{\text {ind }} g^{k}=\sum_{r=0}^{p-2} \epsilon_{r} \alpha^{r}=\sum\left\{\epsilon_{\nu} \alpha^{\nu}+\epsilon_{p^{\prime}+\nu} \alpha^{p^{\prime}+\nu}\right\} \\
& =\sum \epsilon_{\nu} \alpha^{\nu}-\sum\left(1-\epsilon_{\nu}\right) \alpha^{\nu}=2 \sum \epsilon_{\nu} \alpha^{\nu}-\sum \alpha^{\nu} \\
& =2\left\{\sum \epsilon_{\nu} \alpha^{\nu}-1 /(1-\alpha)\right\} .
\end{aligned}
$$

Multiplying by $(1-\alpha)$, we have

$$
(1-\alpha) m_{e}(p)=2 \sum_{n=1}^{p^{\prime}}\left(\epsilon_{n}-\epsilon_{n-1}\right) \alpha^{n}=2 W_{e}(p, 1)
$$

since $\epsilon_{p^{\prime}}=0$. From this the lemma follows.

Lemma 8. $N_{e}\left(m_{e}(p)\right)=N\left(W_{e}(p, 1)\right) 2^{J(e)}$ where

$$
J(e)=\left\{\begin{array}{ll}
\phi(e) & \text { if } e \neq 2^{k} \\
\phi(e)-1 & \text { if } e=2^{k}
\end{array}(k \geqslant 1) .\right.
$$

Proof. This follows at once by taking norms of both sides in Lemma 7. Use is made of a theorem of Lebesgue [4] in writing

$$
\prod_{(t, e)=1}\left(1-\alpha^{t}\right)=Q_{e}(1)=2 \text { or } 1
$$

according as $e$ is a power of an (even) prime or not.

6. Main Theorem. We are now prepared to give a formula for the class number $h^{*}(p)$ as a product of norms of exponential sums of the type $W_{e}(p)$, divided by certain cyclotomic polynomials evaluated at the point 2 . In stating the result there is some recapitulation of notation.

THEOREM 4. Let $p$ be an odd prime with $g$ any primitive root. Let $e$ range over all divisiors of $p-1$ whose codivisors are odd. Let

$$
\tau=\tau(e)=e /\left(e, \text { ind }_{g} 2\right),
$$

and let $h_{e}(p)=p^{[e /(p-1)]} N_{e}\left(W_{e}(p)\right) /\left\{Q_{\tau(e)}(2)\right\}^{\gamma}$, where

$$
\gamma=\gamma(e)=\phi(e) / \phi(\tau)
$$

Then 


$$
h^{*}(p)=\prod_{e} h_{e}(p)
$$

Proof. This follows at once from putting together Theorem 1, Theorem 3, and Lemma 8 , using $e=2^{\lambda} d, \tau=\tau(d)$, and the fact that

$$
\sum_{d \mid \omega} \phi\left(2^{\lambda} d\right)=\frac{p-1}{2}
$$

At first sight, it would appear from (14) that $h^{*}(p)$ is always divisible by $p$. Of course, this is not so. The explanation is that $p$ divides the denominator, $Q_{\tau(\omega)}(2)$. To see this we note [5] that

$$
\tau(\omega)=(p-1) /((p-1), \text { ind } 2)
$$

is the exponent or order of 2 modulo $p$. Hence $p$ is a divisor of $Q_{\tau(\omega)}(2)$. Otherwise, it is the responsibility of the numerator $N$ of each factor to be divisible by the denominator $Q^{\gamma}$. This affords an excellent check on calculation of $N$.

To illustrate Theorem 4 we give the simple example of $p=31$. Here we have $g=3, \lambda=1, \omega=15$, ind $_{3} 2=24$. The various elements in each factor may be tabulated thus.

\begin{tabular}{ccccc}
\hline$e$ & $\tau(e)$ & $\gamma(e)$ & $\left\{Q_{\tau}(2)\right\}^{\gamma}$ & $N_{e}(W)$ \\
\hline 2 & 1 & 1 & 1 & 3 \\
6 & 1 & 2 & 1 & 3 \\
10 & 5 & 1 & 31 & 31 \\
30 & 5 & 2 & $31^{2}$ & 31 \\
\hline
\end{tabular}

Hence

$$
h^{*}(31)=31 \cdot 3 \cdot 3 \cdot \frac{31}{31} \cdot \frac{31}{31^{2}}=9 .
$$

\begin{tabular}{|c|c|c|}
\hline$\delta$ & $\tau$ & $\gamma$ \\
\hline 1 & $e$ & 1 \\
\hline 2 & $e / 2$ & $\begin{cases}1 & \text { if } 2 \| e \\
2 & \text { otherwise }\end{cases}$ \\
\hline 4 & $e / 4$ & $\begin{cases}2 & \text { if } 4 \| e \\
4 & \text { otherwise }\end{cases}$ \\
\hline$q$ & $e / q$ & $\left\{\begin{array}{cl}q-1 & \text { if } q \| e \\
q & \text { otherwise }\end{array}\right.$ \\
\hline $2 q$ & $e /(2 q)$ & $\left\{\begin{aligned} 2 & \text { if } 2\|e, q\| e \\
q & \text { if } 2 \| e, q^{2} \mid e \\
2 q-2 & \text { if } 4 \mid e, q \| e \\
2 q & \text { otherwise }\end{aligned}\right.$ \\
\hline
\end{tabular}

7. Simple Special Cases. When the greatest common divisor $\left(2^{\lambda} d\right.$, ind 2$)=\delta$, is specified, the parameters $\tau$ and $\gamma$ can be tabulated as follows. Here we have written $e$ for $2^{\lambda} d$ and $q$ is an odd prime. 
The case where $p$ is a Fermat prime results in (14) having but a single factor. Setting $p=2^{2^{\nu}}+1$, we find $q=3, \lambda=2^{\nu}, \omega=1, e=2^{2^{\nu}}$, so $\tau(e)=2^{\nu+1}, \gamma(e)=$ $2^{2^{\nu}-\nu-1}, Q_{\tau}(2)=2^{2^{\nu}}+1=p$. For example, for $p=257$ we have $\nu=3$ so $\gamma(e)=$ 16. This means that $N_{256}\left(W_{256}(257)\right)$ must be divisible by $257^{15}$ and since 257 is an irregular prime, we can expect $257^{16}$. In fact,

$$
\begin{aligned}
h^{*}(257)= & 257 \cdot 20738946049 . \\
& 1022997744563911961561298698183419037149697
\end{aligned}
$$

a factorization into primes.

This alarmingly large value of $\gamma$ is unusual for primes $p$ in general. Ordinarily, $\gamma$ rarely exceeds 2 and the denominator $Q^{\gamma}$ is very small compared with the numerator $N(W)$ in (14).

8. Odd Intrinsic Factors of $h_{e}(p)$. For those odd primes $q$ which divide both $e$ and $h_{e}(p)$ there is a "Taw of repetition", namely

THEOREM 5. Let $p-1=$ ef where $f$ is odd. Let $q$ be a prime factor of $f$. Then $h_{e q}(p)$ is divisible by $q$ if and only if $h_{e}(p)$ is divisible by $q$.

Proof. By (12) and (8) it suffices to prove the same fact about $Q_{e q}^{*}$ and $Q_{e}^{*}$.

Now

$$
Q_{e q}^{*}=N_{e q}\left(M_{e q}(p)\right)=\prod_{(t, e q)=1 ; t<e q} \sum_{n=1}^{q-1} g_{n} \alpha_{1}^{t n}
$$

where we have set $\alpha_{1}=\exp \{2 \pi i /(e q)\}$ so that $\alpha_{1}^{q}=\alpha$. If we use the multinomial theorem identity

$$
\left(x_{1}+x_{2}+\cdots+x_{p-1}\right)^{q}=x_{1}^{q}+x_{2}^{q}+\cdots+x_{p-1}^{q}+q F\left(x_{1}, \ldots, x_{p-1}\right),
$$

we have

$$
\left(Q_{e q}^{*}\right)^{q}=\prod_{(t, e q)=1} \sum_{n=1}^{q-1} g_{n}^{q} \alpha^{t n}+q \Phi
$$

where $\Phi$ is a symmetric polynomial in the powers of $\alpha_{1}$ with integer coefficients.

Thus we have

$$
Q_{e q}^{*} \equiv\left\{\prod_{t \leqslant e ;(t, e)=1} \sum_{n=1}^{p-1} g_{n} \alpha^{t n}\right\}^{\phi(e q) / \phi(e)}(\bmod q)
$$

or

$$
Q_{e q}^{*} \equiv\left(Q_{e}^{*}\right)^{\theta} \quad(\bmod q)
$$

where

$$
\theta= \begin{cases}1 & \text { if } q \mid e \\ q-1 & \text { otherwise }\end{cases}
$$

Thus $q \mid Q_{e q}^{*}$ if and only if $q \mid Q_{e}^{*}$. This proves the theorem. 
Example. Take $p=379, p-1=2 \cdot 3^{3} \cdot 7$. Here $3 \mid h_{2}=3$. Hence $3 \mid h_{6}=$ $3 \cdot 13,3 \mid h_{18}=3 \cdot 991$ and $3 \mid h_{54}=3 \cdot 29997973$. This theorem includes a theorem of Metsänkylä [6] for $e=2^{\lambda}$.

9. The Intrinsic Factor 2 . It is well known that for $p \equiv 3(\bmod 4), h_{2}(p)$ is always odd. For $e \neq 2$, however, $h_{e}(p)$ can be even, as witness

$$
h_{28}(29)=8, \quad h_{6}(163)=4, \quad h_{14}(491)=2^{6} \cdot 29 .
$$

Newman [8] conjectured and Metsänkylä [6] proved that if $h_{p}^{*}$ is even it is a multiple of 4 . The latter's results show that when $e=2^{\lambda}, h_{e}(p)$ is odd and that when $e=2^{\lambda} d$ with $d>1$ then the highest power of 2 dividing $h_{e}(p)$ is $2^{j \nu}$ where $\nu$ is the exponent of $2(\bmod d)$ and $j \geqslant 0$. Since $\nu \geqslant 2$, Newman's conjecture follows at once. That $j$ can be greater than 1 is evidenced by

$$
h_{62}(311)=2^{10} \cdot 9918966461,
$$

whereas the exponent of $2(\bmod 31)$ is 5 . Since

$$
2^{j v} \equiv 1 \quad(\bmod d)
$$

the factor $2^{j \nu}$ of $h_{e}(p)$ behaves somewhat like a characteristic prime power factor of $h_{e}(p)$, being of the form $d x+1$ rather than $2^{\lambda} d x+1$.

10. Application. The preceding results have been used to obtain the prime factorization of $h^{*}(p)$ in the published tables of Newman [8] $(p<200)$ and Schrutka [7] $(p \leqslant 257)$ and in the as yet unpublished table of Lehmer and Masley [9] $(p<$ 512). Computational methods and results will appear in [9].

Department of Mathematics

University of California, Berkeley

Berkeley, California 94720

1. E. KUMMER, "Bestimmung der Anzahl nicht äquivalenter Classen für die aus $\lambda$ ten Wurzeln der Einheit gebildeten complexen Zahlen und die idealen Factoren derselben," J. Reine Angew. Math., v. 40, 1850, pp. 43-116 (p. 110, formula 38).

2. D. H. LEHMER, "Factorization of certain cyclotomic functions," Ann. of Math. (2) v. 34, 1933, pp. $461-479$ (p. 463, Theorem 3).

3. Z. I. BOREVICH (BOREVIČ) \& I. R. SHAFAREVICH (ک̌AFAREVIČ), Number Theory, "Nauka", Moscow, 1964; English transl., Pure and Appl. Math., vol. 20, Academic Press, New York, 1966. MR 30 \#1080; 33 \#4001.

4. V. A. LEBESGUE, "Dimostrazione dell'irreduttibilitá dell'equazione formata con le radici primitive dell'unitá," Ann. Mat. Pura Appl., v. 2, 1859, pp. 232-237.

5. W. J. LEVEQUE, Topics in Number Theory, Vol. 1, Addison-Wesley, Reading, Mass., 1956, p. 48 (Theorem 4.1).. MR 18, 283.

6. T. METSÄNKYLÄ, "On prime factors of the relative class numbers of cyclotomic fields," Ann. Univ. Turku. Ser. A I, No. 149, 1971,8 pp. MR 44 \#178.

7. G. SCHRUTKA V. RECHTENSTAMM, "Tabelle der (Relativ)-Klassenzahlen der Kreiskörper, deren $\phi$-Funktion des Wurzelexponenten (Grad) nicht grösser als 256 ist," Abh. Deutsch. Akad. Wiss. Berlin Kl. Math. Phys. Tech., 1964, no. 2, 64 pp. MR 29 \#4918.

8. M. NEWMAN, "A table of the first factor for prime cyclotomic fields," Math. Comp., v. 24, 1970, pp. 215-219. MR 41 \#1684.

9. D. H. LEHMER \& J. M. MASLEY, "Table of the cyclotomic class numbers $h^{*}(p)$ and their factors for $200<p<512$." (To appear.) 